\title{
Role of energy correlations on Coulomb suppression of shot noise in ballistic conductors contacted to degenerate reservoirs
}

\author{
T. González, J. Mateos, and D. Pardo \\ Departamento de Física Aplicada, Universidad de Salamanca, Plaza de la Merced, s/n, E-37008 Salamanca, Spain \\ G. Gomila \\ Departament d'Electrònica and CBEN, Universitat de Barcelona, C/ Marti i Franqués, 1, E-08028 Barcelona, Spain \\ I. R. Cantalapiedra \\ Departament de Física Aplicada, Universitat Politècnica de Catalunya, Av. Dr. Marañon, 44, E-08028 Barcelona, Spain \\ L. Reggiani \\ INFM-National Nanotechnology Laboratory and Dipartimento di Ingegneria dell'Innovazione, Universitá di Lecce, Via Arnesano s/n, \\ I-73100 Lecce, Italy
}

(Received 23 January 2003; published 14 August 2003)

\begin{abstract}
Coulomb suppression of shot noise in a ballistic diode connected to degenerate ideal contacts is analyzed in terms of the correlations taking place between current fluctuations due to carriers injected with different energies. By using Monte Carlo simulations we show that at low frequencies the origin of Coulomb suppression can be traced back to the negative correlations existing between electrons injected with an energy close to that of the potential barrier present in the diode active region and all other carriers injected with higher energies. Correlations between electrons with energy above the potential barrier with the rest of electrons are found to influence significantly the spectra at high frequency in the cutoff region.
\end{abstract}

DOI: 10.1103/PhysRevB.68.075309

PACS number(s): 72.70. $+\mathrm{m}, 73.23 .-\mathrm{b}, 73.50 . \mathrm{Td}, 05.40 . \mathrm{Ca}$

\section{INTRODUCTION}

The systematic trend of downsizing electronic devices (such as the channel length of field-effect transistors) has led to the design and fabrication of structures exhibiting electron ballistic transport, ${ }^{1-3}$ even at room temperature. ${ }^{4,5}$ For the potential application of these devices, the achievement of a low noise level is of primary importance. ${ }^{6}$ For this reason, the analysis of shot-noise suppression in multimode ballistic conductors has received renewed attention in recent years. ${ }^{6-9,11-13}$

In the study of these structures it has been shown that two fundamental physical interactions can contribute to suppress shot noise. On the one hand, the current fluctuations originated by the thermal emission of carriers from the contacts acting as reservoirs may carry the signature of Pauli correlations. ${ }^{14}$ On the other hand, these fluctuations can be modified by the action of long-range Coulomb interaction in the ballistic region. ${ }^{9,10}$ Recently, some of the authors ${ }^{13}$ developed an analytical theory describing the relative relevance of these suppression mechanisms and validated it by comparison with numerical Monte Carlo (MC) simulations. Conditions corresponding to all physically relevant conditions where analyzed: from nondegenerate to degenerate injection, from short to long samples, and from low to high applied voltages.

In spite of these efforts, a truly microscopic understanding of the nature of shot noise suppression in ballistic devices, and in particular of Coulomb suppression, is still lacking in the current literature. Indeed, while the correlations induced by Pauli exclusion principle and the related shot-noise suppression are well understood on a microscopic basis, ${ }^{14-16}$ the case of Coulomb correlations has been less explored. Typically, Coulomb suppression is generally ascribed to the $a c$ tion of the self-consistent fluctuations of the potential barrier present in the active region of the structures, which smooth out the current fluctuations imposed by the random injection of electrons at the contacts. ${ }^{7,9,10}$ Bulashenko et al., in Refs. 9,17 , further proposed a microscopic interpretation of shot noise suppression in terms of energy correlations by introducing, in a formal way, the correlation between current fluctuations associated with carriers injected with different energies. However, a quantitative evaluation of this interesting quantity has not yet been provided, so that a detailed investigation of the role played by energy correlations on Coulomb suppression is still not available. The aim of this work is precisely to address this issue. By means of MC simulations, the role played by electrons injected with different energy levels on the onset of Coulomb suppression is determined. To this end, the correlation spectra between the contributions to the current fluctuations originating from carriers injected with different energies are evaluated. The results indicate that Coulomb shot-noise suppression is originated by the negative energy correlation spectra at low frequency taking place between the carriers injected with an energy close to that of the potential barrier and all other carriers injected with higher energies. Interestingly, we have found the emergence of correlations between high-energy electrons at high frequencies near the cutoff region whose nature has also been analyzed.

The paper is organized as follows. In Sec. II we describe the physical model used in the calculations and the structure under study. The effects of Fermi and Coulomb suppression on the shot noise exhibited by the structure are analyzed in 


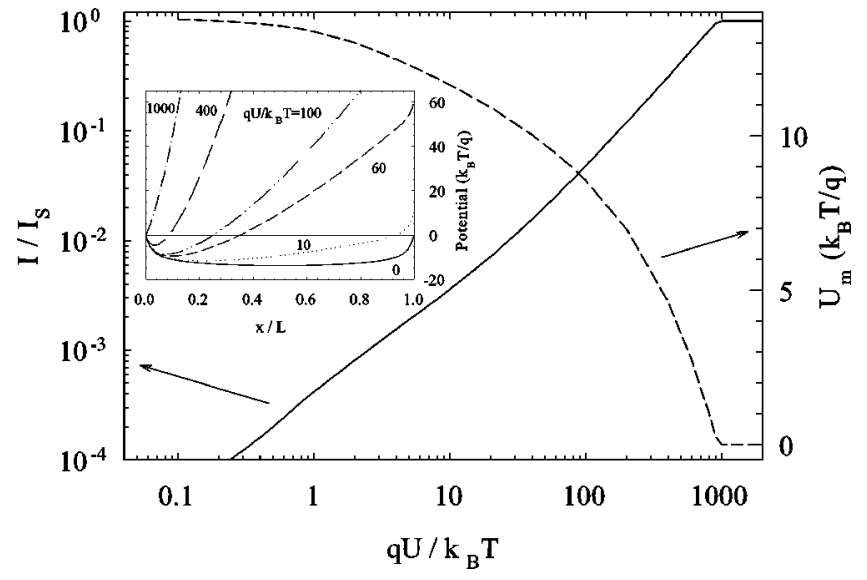

FIG. 1. Current [normalized to the saturation value $I_{S}$ $\left.=\int_{0}^{\infty} I_{C}\left(\varepsilon_{x}\right) d \varepsilon_{x}\right]$ and amplitude of the potential minimum vs applied voltage (normalized to the thermal value). The inset shows the spatial profile of the potential $q \phi(x) / k_{B} T$ for different applied voltages.

Sec. III. In Sec. IV the analytical expressions associated with the energy correlation spectra are derived, and the corresponding MC results are reported and used to analyze the role of energy correlations on the Coulomb suppression of shot noise. Finally, in Sec. V the main conclusions of the paper are summarized.

\section{PHYSICAL MODEL AND SIMULATED STRUCTURE}

For the calculations, we consider a structure consisting of a lightly doped active region of length $L$ and cross-sectional area $A$ sandwiched between two heavily doped (degenerate) regions of the same material which act as ideal injecting contacts. The structure is assumed to be sufficiently thick in transversal directions to allow a 1D electrostatic treatment. Thus, the simulation is $1 \mathrm{D}$ in real space and $3 \mathrm{D}$ in momentum space. The contacts are characterized by a carrier density $n_{c}$ and the associated Fermi level $\varepsilon_{F}$. Once injected with a given energy, electrons move ballistically inside the sample. Therefore, noise originates from the randomness in time of electron injection from the contacts, that is modeled in accordance with Fermi statistics as described in Refs. 8 and 14. In the calculations we use the following parameters, which correspond to the possible realistic case of a GaAs diode: lattice temperature $T=77 \mathrm{~K}$, effective mass $m=0.065 m_{0}$ ( $m_{0}$ being the free electron mass), dielectric constant $\epsilon$ $=12.9 \epsilon_{0}$ ( $\epsilon_{0}$ being the vacuum permitivity), $L=300 \mathrm{~nm}$, and $n_{c}=1.3 \times 10^{18} \mathrm{~cm}^{-3}\left(\varepsilon_{F}=10 k_{B} T\right)$. These values lead to $L / L_{0}=20.23,{ }^{13}$ with $L_{0}$ the Debye length associated with the effective density of states in the conduction band of the semiconductor. According to the theory developed in Ref. 13, the case $L / L_{0}=20.23$ and $\varepsilon_{F} / k_{B} T=10$ corresponds to a diode where both Pauli and Coulomb correlations are present.

The current-voltage $(I-U)$ characteristic of the diode, with the current normalized to the saturation value $I_{S}$ and the voltage to the thermal value $k_{B} T / q$, is reported in Fig. 1. At low voltages a linear behavior is found, while for high voltages near saturation a superlinear behavior close to a $U^{3 / 2}$ dependence is observed (space-charge limited conditions). The inset of Fig. 1 shows the potential profile along the structure for different applied voltages. This profile exhibits the characteristic minimum (energy barrier) decreasing in amplitude and shifting towards the cathode as $U$ increases. The amplitude of the energy barrier $q U_{m}$ is reported in the main figure as a function of the applied voltage. For the diode considered here, $U_{m}=13.82 k_{B} T / q$ for $U=0$ and $U_{m}$ $=0$ for $U=U_{\mathrm{sat}} \approx 950 k_{B} T / q$.

\section{FERMI AND COULOMB SUPPRESSION}

The Monte Carlo technique, by allowing the use of static (frozen potential profile) and dynamic (potential profile updated at each time step) simulation schemes, offers a unique possibility to evidence the effect of Coulomb interaction on shot-noise suppression. ${ }^{7}$ Thus, when static simulations are performed, no Coulomb correlation among carriers takes place in the active region, and the only source of suppression is the Fermi statistics associated with electrons at the contacts. This allows calculating the Fermi shot-noise suppression factor as $\gamma_{F}=S_{I}^{S}(0) / 2 q I$, where $S_{I}^{S}(0)$ is the lowfrequency static current spectral density and $I$ the electric current. An analytical expression for $\gamma_{F}$ can be easily derived by using that the low frequency static current spectral density and electric current are given by

$$
S_{I}^{S}(0)=\int_{q U_{m}}^{\infty} S_{I_{c}}\left(\varepsilon_{x}\right) d \varepsilon_{x}+\int_{q\left(U_{m}+U\right)}^{\infty} S_{I_{c}}\left(\varepsilon_{x}\right) d \varepsilon_{x}
$$

and

$$
I=\int_{q U_{m}}^{\infty} I_{c}\left(\varepsilon_{x}\right) d \varepsilon_{x}-\int_{q\left(U_{m}+U\right)}^{\infty} I_{c}\left(\varepsilon_{x}\right) d \varepsilon_{x},
$$

respectively. Here, $q U_{m}$ is the amplitude of the energy barrier (known from the simulations), $U$ the applied voltage, $\varepsilon_{x}$ the longitudinal kinetic energy, $I_{c}\left(\varepsilon_{x}\right) d \varepsilon_{x}$ the current associated with electrons injected with energy between $\varepsilon_{x}$ and $\varepsilon_{x}$ $+d \varepsilon_{x}$ at the contacts, and $S_{I_{c}}\left(\varepsilon_{x}\right) d \varepsilon_{x}$ the low-frequency spectral density of the fluctuations of such a current. The explicit expressions for these quantities $\operatorname{read}^{6,8,11}$

$$
\begin{aligned}
& I_{c}\left(\varepsilon_{x}\right)=\frac{q m A}{2 \pi^{2} \hbar^{3}} \int_{0}^{\infty} f\left(\varepsilon_{x}+\varepsilon_{t}\right) d \varepsilon_{t} \\
& =\frac{q m A k_{B} T}{2 \pi^{2} \hbar^{3}} \ln \left[1+\exp \left(\frac{\varepsilon_{F}-\varepsilon_{x}}{k_{B} T}\right)\right], \\
& S_{I_{c}}\left(\varepsilon_{x}\right)=2 q \frac{q m A}{2 \pi^{2} \hbar^{3}} \int_{0}^{\infty} f\left(\varepsilon_{x}+\varepsilon_{t}\right)\left[1-f\left(\varepsilon_{x}+\varepsilon_{t}\right)\right] d \varepsilon_{t} \\
& =2 q k_{B} T \frac{\partial I_{c}\left(\varepsilon_{x}\right)}{\partial \varepsilon_{F}}=\frac{q^{2} m A k_{B} T}{\pi^{2} \hbar^{3}} f\left(\varepsilon_{x}\right),
\end{aligned}
$$

where $\varepsilon_{t}$ is the transversal electron energy and $f(\varepsilon)=\{1$ $\left.+\exp \left[\left(\varepsilon-\varepsilon_{F}\right) / k_{B} T\right]\right\}^{-1}$ the thermal equilibrium Fermi-Dirac distribution. The suppression effect associated with Pauli 


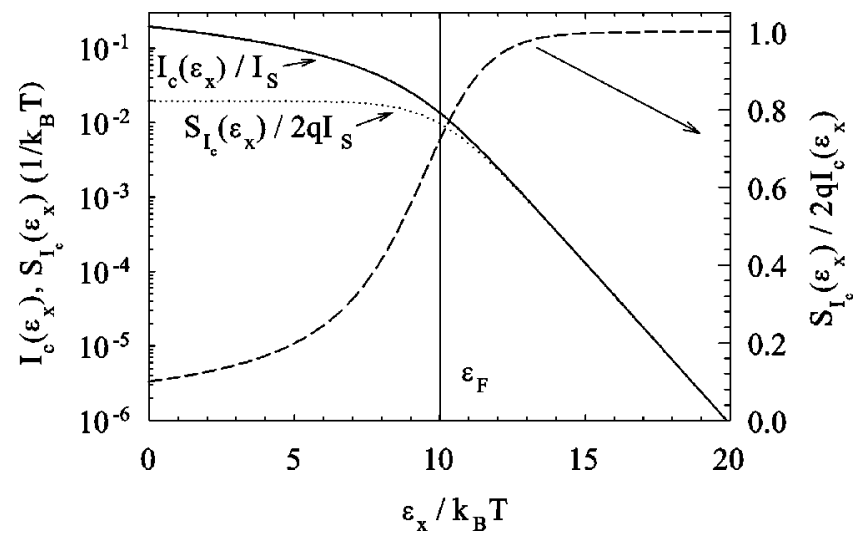

FIG. 2. Current $I_{C}\left(\varepsilon_{x}\right)$ (normalized to the saturation value $I_{S}$ ) and low-frequency noise $S_{I_{C}}\left(\varepsilon_{x}\right)$ (normalized to $2 q I_{S}$ ) contributions of the injected electrons as a function of the longitudinal energy $\varepsilon_{x}$ (left scale), and ratio between both quantities (right scale).

correlations takes place through the injecting statistics and is clearly observed in Fig. 2, which shows $I_{c}\left(\varepsilon_{x}\right)$ and $S_{I_{c}}\left(\varepsilon_{x}\right)$ as a function of $\varepsilon_{x}$. Carriers injected with $\varepsilon_{x} \gg \varepsilon_{F}$ obey Poissonian statistics and exhibit full shot noise $S_{I_{c}}\left(\varepsilon_{x}\right)$ $=2 q I_{c}\left(\varepsilon_{x}\right)$. By contrast, carriers injected with $\varepsilon_{x} \ll \varepsilon_{F}$ exhibit a significantly suppressed shot noise $S_{I_{c}}\left(\varepsilon_{x}\right)$ $<2 q I_{c}\left(\varepsilon_{x}\right)$ with a suppression ratio approaching

$$
\frac{S_{I_{c}}\left(\varepsilon_{x}\right)}{2 q I_{c}\left(\varepsilon_{x}\right)} \sim \frac{k_{B} T}{\varepsilon_{F}-\varepsilon_{x}} .
$$

Closed circles in Fig. 3 report the MC results for $\gamma_{F}$ as a function of both the applied voltage $U$, Fig. 3(a), and the amplitude of the potential minimum $U_{m}$, Fig. 3(b). The theoretical values calculated from the analytical expression derived by means of Eqs. (1) and (2) (solid line) perfectly agree with the static simulations, as should be. We note that at low $U$, when $q U<k_{B} T$, thermal noise is dominant and $\gamma_{F}>1$. For intermediate values of $U$, when $k_{B} T<q U$ and $q U_{m}$ $>\varepsilon_{F}, I$ is determined by Poissonian electrons with $\varepsilon_{x}>\varepsilon_{F}$ in the tail of the Fermi distribution (low-occupation states), and thus $\gamma_{F}=1$. At higher $U$, when $q U_{m}<\varepsilon_{F}$, the contribution to $I$ of carriers injected with sub-Poissonian statistics becomes more and more important. Accordingly, $\gamma_{F}$ decreases systematically taking values smaller than unity, until saturating for $U>U_{\text {sat }}\left(q U_{m}=0\right)$ at the value $\gamma_{F}$ $=2 k_{B} T / \varepsilon_{F} \cdot{ }^{11,13}$

When dynamic simulations are performed, the temporal fluctuations of the electric field modulate the transmission of electrons over the potential minimum, thus modifying the noise characteristics with respect to the static case. Now, in addition to Fermi suppression, a further reduction of noise due to Coulomb correlations takes place. Since the two suppression mechanisms act independently, the low-frequency dynamic spectral density $S_{I}^{d}(0)$ can be expressed as $S_{I}^{d}(0)$ $=\gamma 2 q I=\gamma_{F} \gamma_{C} 2 q I$, where $\gamma=\gamma_{F} \gamma_{C}$ is the total shot-noise suppression factor and $\gamma_{C}$ the Coulomb suppression factor. ${ }^{8,11-13}$ In Fig. 3 we present the calculated values of the total suppression factor as obtained from dynamic MC simu-
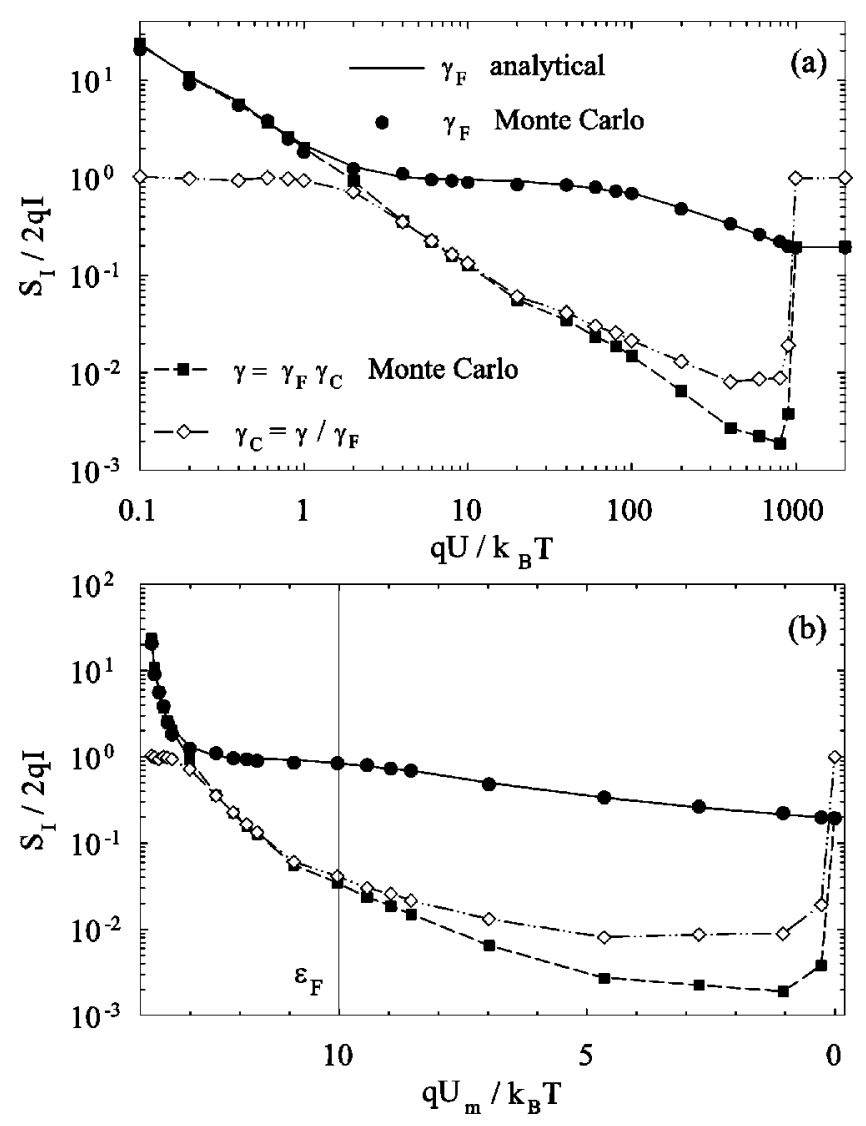

FIG. 3. Total suppression (Fano) factor $\gamma$ (calculated with static and dynamic simulation schemes), and Coulomb $\gamma_{C}$ and Fermi $\gamma_{F}$ suppression factors as a function of (a) applied voltage $U$ and (b) amplitude of the potential minimum $U_{m}$.

lations (closed squares) and the corresponding factorization into Coulomb (open diamonds) and Fermi (closed circles) contributions. As can be observed, at the lowest values of $U$, thermal noise is dominant, and thus $S_{I}^{d}(0)=S_{I}^{s}(0)$ and $\gamma_{C}$ $\approx 1$ (i.e., absence of Coulomb suppression). Then, at increasing $U$, three different regimes of suppression are identified. The first regime $\left(k_{B} T<q U, q U_{m}>\varepsilon_{F}\right)$ is related only to Coulomb correlations $\left(\gamma_{F}=1, \gamma_{C}<1\right)$. Here $I$ is due to Poissonian carriers (with $\varepsilon_{x}>\varepsilon_{F}$ ) which give rise to fluctuations of $U_{m}$ modulating the passage of further electrons, thus leading to increasing Coulomb suppression. The second regime $\left(q U_{m}<\varepsilon_{F}\right)$ is related to both Coulomb and Fermi correlations $\left(\gamma_{F}<1, \gamma_{C}<1\right)$. Here, interestingly, $\gamma_{C}$ tends to saturate while $\gamma_{F}$ keeps decreasing with $U$. This is due to the fact that, as $U$ increases, the additional carriers contributing to the current come from the energy region below $\varepsilon_{F}\left(\varepsilon_{x}<\varepsilon_{F}\right.$, characterized by sub-Poissonian statistics) and prevent further significant fluctuations of $U_{m}$. Finally, in the third regime (under current saturation) the suppression is constant and only due to Fermi correlations $\left(\gamma_{F}=2 k_{B} T / \varepsilon_{F}, \gamma_{C}=1\right)$.

\section{ENERGY CORRELATIONS IN COULOMB SUPPRESSION}

Within our model, carriers injected at the contacts with different $\varepsilon_{x}$ levels are uncorrelated. ${ }^{11-13}$ However, electrons 
with different energies in the volume of the conductor are expected to be strongly correlated by Coulomb interaction. To investigate how these correlations, responsible for Coulomb suppression, take place, we will calculate them by means of MC simulations.

In order to simplify the discussion, we will consider the particularly favorable situation in which $\varepsilon_{F} \ll q U<q U_{\text {sat }}$. In this case Coulomb suppression is very significant (see Fig. 3 ), and the average current $I$ and the low-frequency current spectral density $S_{I}(0)$ are determined only by carriers injected at the left contact. The contribution to the instantaneous current coming from electrons injected at the right contact can be neglected, since they rapidly turn back to the same contact. Thus, the instantaneous value of the electric current can be written as

$$
I(t)=\int_{0}^{\infty} I_{L}\left(\varepsilon_{x}, t\right) d \varepsilon_{x},
$$

where $I_{L}\left(\varepsilon_{x}, t\right) d \varepsilon_{x}$ is the instantaneous current due to electrons injected with $\varepsilon_{x}$ at the left contact, a quantity that can be easily evaluated from MC simulations. The fluctuations of the current are then given by

$$
\delta I(t)=\int_{0}^{\infty} \delta I_{L}\left(\varepsilon_{x}, t\right) d \varepsilon_{x}=\int_{0}^{\infty}\left[I_{L}\left(\varepsilon_{x}, t\right)-\bar{I}_{L}\left(\varepsilon_{x}\right)\right] d \varepsilon_{x},
$$

where $\bar{I}_{L}\left(\varepsilon_{x}\right) d \varepsilon_{x}$ is the time-average value of $I_{L}\left(\varepsilon_{x}, t\right) d \varepsilon_{x}$. The autocorrelation function of current fluctuations can then be evaluated as

$$
\begin{aligned}
C_{I}(t) & =\overline{\delta I\left(t^{\prime}\right) \delta I\left(t^{\prime}+t\right)} \\
& =\int_{0}^{\infty} \int_{0}^{\infty} \overline{\delta I_{L}\left(\varepsilon_{x}, t^{\prime}\right) \delta I_{L}\left(\varepsilon_{x}^{\prime}, t^{\prime}+t\right)} d \varepsilon_{x} d \varepsilon_{x}^{\prime} \\
& =\int_{0}^{\infty} \int_{0}^{\infty} C_{I_{L}}\left(\varepsilon_{x}, \varepsilon_{x}^{\prime}, t\right) d \varepsilon_{x} d \varepsilon_{x}^{\prime},
\end{aligned}
$$

where $C_{I_{L}}\left(\varepsilon_{x}, \varepsilon_{x}^{\prime}, t\right)$ is the correlation function between current fluctuations due to carriers injected with energies $\varepsilon_{x}$ and $\varepsilon_{x}^{\prime}$ at the left contact, quantity that can be readily calculated from MC simulations. The current spectral density can then be expressed as

$$
\begin{aligned}
S_{I}(f) & =2 \int_{-\infty}^{\infty} C_{I}(t) \exp (j 2 \pi f t) d t \\
& =\int_{0}^{\infty} \int_{0}^{\infty} S_{I_{L}}\left(\varepsilon_{x}, \varepsilon_{x}^{\prime}, f\right) d \varepsilon_{x} d \varepsilon_{x}^{\prime},
\end{aligned}
$$

with

$$
S_{I_{L}}\left(\varepsilon_{x}, \varepsilon_{x}^{\prime}, f\right)=2 \int_{-\infty}^{\infty} C_{I_{L}}\left(\varepsilon_{x}, \varepsilon_{x}^{\prime}, t\right) \exp (j 2 \pi f t) d t .
$$

Therefore, $S_{I_{L}}\left(\varepsilon_{x}, \varepsilon_{x}^{\prime}, f\right)$, representing the spectrum of the correlation between current fluctuations due to carriers in-

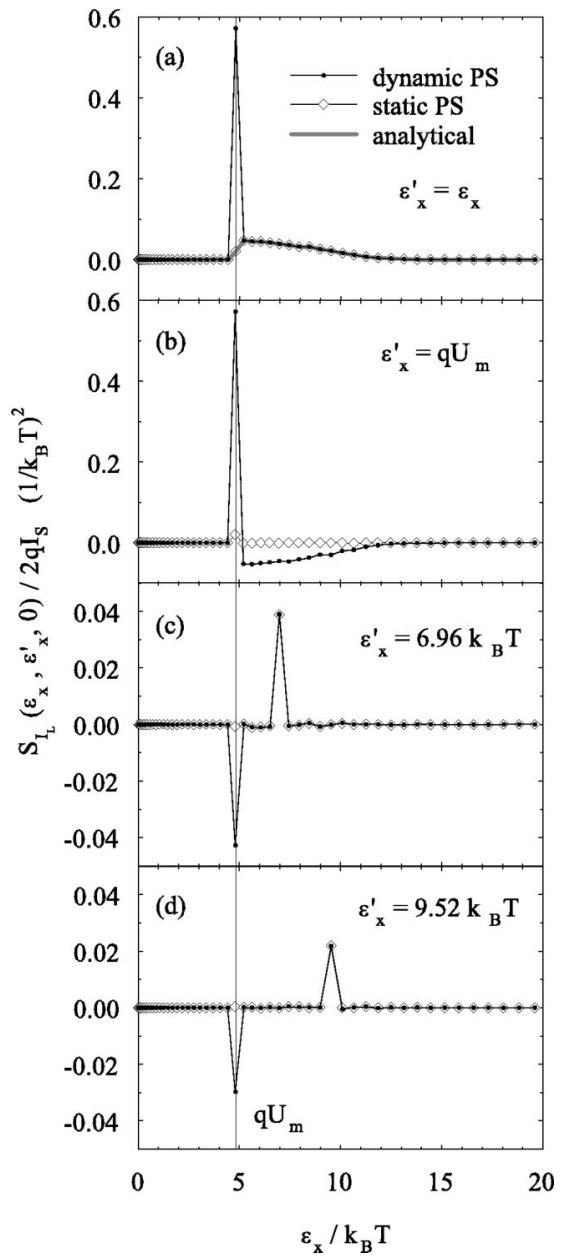

FIG. 4. Low-frequency value of the correlation spectra between current fluctuations due to electrons injected with energies $\varepsilon_{x}$ and $\varepsilon_{x}^{\prime}$ at the left contact as a function of $\varepsilon_{x}$ for several values of $\varepsilon_{x}^{\prime}$ : (a) $\varepsilon_{x}^{\prime}=\varepsilon_{x}$ (diagonal contributions), (b) $\varepsilon_{x}^{\prime}=q U_{m}$, (c) $\varepsilon_{x}^{\prime}=6.96 k_{B} T$, and (d) $\varepsilon_{x}^{\prime}=9.52 k_{B} T . q U / k_{B} T=380$. Vertical lines indicate the position of $q U_{m}=4.80 k_{B} T$. Closed circles and open diamonds correspond, respectively, to the results obtained with dynamic and static simulations. The gray line in (a) is the analytical expectation for the static case.

jected with different values of longitudinal energy $\varepsilon_{x}$ and $\varepsilon_{x}^{\prime}$ at the left contact, is the key quantity to evaluate (and identify) the correlations induced by Coulomb interaction inside the active region. To illustrate the properties of this quantity, we have considered the case of $U=380 k_{B} T / q$, for which the energy barrier is $q U_{m}=4.80 k_{B} T$ and a significant Coulomb suppression takes place. To perform the calculations indicated in Eqs. (7)-(10), in MC simulations it is necessary to discretize the range of the longitudinal energies $\varepsilon_{x}$ under consideration. In our case the range of $\varepsilon_{x}$ from 0 to $20 k_{B} T$ has been divided into 50 nonuniform meshes. ${ }^{18}$

We discuss first the low-frequency results. Figure 4 reports $S_{I_{L}}\left(\varepsilon_{x}, \varepsilon_{x}^{\prime}, 0\right)$ as a function of $\varepsilon_{x}$ for different values of $\varepsilon_{x}^{\prime}$, with Fig. 4(a) showing the diagonal contribution $\left(\varepsilon_{x}\right.$ $=\varepsilon_{x}^{\prime}$ ) and Figs. 4(b), 4(c), and 4(d) the off-diagonal contributions at energies $\varepsilon_{x}^{\prime}$ equal and above the top of the energy barrier $q U_{m}$. We note that, when the effect of space charge 
is neglected (static simulation), electrons in the active region are uncorrelated and thus only the diagonal contributions at energies above the barrier are expected to differ from zero, $S_{I_{L}}^{s}\left(\varepsilon_{x}, \varepsilon_{x}^{\prime}, 0\right)=S_{I_{c}}\left(\varepsilon_{x}\right) \delta\left(\varepsilon_{x}-\varepsilon_{x}^{\prime}\right) \theta\left(\varepsilon_{x}-q U_{m}\right)$, where $\theta$ is the Heaviside step function and $S_{I_{c}}\left(\varepsilon_{x}\right)$ is given by Eq. (4), corresponding to the noise associated with the Fermi-Dirac injecting statistics. This is confirmed by the static MC results. Indeed, Fig. 4(a) shows an excellent agreement between the results of MC simulations and the analytical values. In the case of Figs. 4(b)-4(d), the only correlation which differs from zero is confirmed to be the diagonal (autocorrelation) contribution, that is, for $\varepsilon_{x}=\varepsilon_{x}^{\prime}$. As indicated by Eq. (1), the integration of these diagonal contributions over $\varepsilon_{x}$ provides the value of the low-frequency static spectral density, containing only the influence of Pauli correlations.

The origin of Coulomb suppression can be understood in terms of the significant differences which appear in the energy correlations with respect to the static case when dynamic simulations are performed. As observed in Figs. 4(c) and 4(d), carriers injected with $\varepsilon_{x}>q U_{m}$, apart from the autocorrelation (positive peak) associated with their injecting statistics already observed in the static case, are only (anti) correlated (negative peak) with those electrons injected with $\varepsilon_{x} \approx q U_{m}$. This indicates that a positive fluctuation of $I_{L}\left(\varepsilon_{x}, t\right)$ at $\varepsilon_{x}>q U_{m}$ induces, via Coulomb interaction (barrier fluctuations), a negative fluctuation of $I_{L}\left(q U_{m}, t\right)$, and vice versa. Thus, the terms $S_{I_{L}}^{d}\left(\varepsilon_{x}, \varepsilon_{x}^{\prime}, 0\right)$ being different from zero are (i) the diagonal contributions $S_{I_{L}}^{d}\left(\varepsilon_{x}, \varepsilon_{x}, 0\right)$, for $\varepsilon_{x}$ $\geqslant q U_{m}$, which are positive [see Fig. 4(a)] and (ii) the offdiagonal contributions involving carriers at $\varepsilon_{x} \approx q U_{m}$, that is, $S_{I_{L}}^{d}\left(\varepsilon_{x}, q U_{m}, 0\right)$ for $\varepsilon_{x}>q U_{m}$ [see Fig. 4(b)] and $S_{I_{L}}^{d}\left(q U_{m}, \varepsilon_{x}^{\prime}, 0\right)$ for $\varepsilon_{x}^{\prime}>q U_{m}$ [see Figs. 4(c) and 4(d)], which are negative. By virtue of the symmetry properties of $C_{I_{L}}\left(\varepsilon_{x}, \varepsilon_{x}^{\prime}, t\right) \quad\left[\right.$ in particular, $C_{I_{L}}\left(\varepsilon_{x}, \varepsilon_{x}^{\prime}, t\right)=C_{I_{L}}\left(\varepsilon_{x}^{\prime}, \varepsilon_{x}\right.$, $-t)], S_{I_{L}}^{d}\left(\varepsilon_{x}, q U_{m}, 0\right)=S_{I_{L}}^{d}\left(q U_{m}, \varepsilon_{x}, 0\right)$. These negative (anticorrelation) terms, by reducing the value of $S_{I}^{d}(0)$ when performing the integration in Eq. (9), are responsible for the noise suppression. The anticorrelation is larger the lower $\varepsilon_{x}$ is [see Fig. 4(b)], since the injection rate and the associated noise are higher (see Fig. 2). The carriers injected with $\varepsilon_{x}$ $\approx q U_{m}$ are those which feel (at low frequency) the influence of the barrier fluctuations caused by the electrons with sufficient energy to overcome the barrier. These barrier fluctuations tend to bunch the carriers injected with $\varepsilon_{x} \approx q U_{m}$ when passing over (or being reflected at) the barrier, thus leading to positive correlations among them. As a consequence, these carriers are found to be strongly autocorrelated when compared to the static case, ${ }^{9}$ as observed in Figs. 4(a) and (b).

We remark that even if some qualitative predictions about the behavior of low-frequency carrier correlations in ballistic diodes can be found in the literature, ${ }^{9,17}$ here these correlations are evidenced and evaluated quantitatively. Moreover, the other advantage of our MC approach is that highfrequency correlations can also be calculated. We analyze some of their properties in the following.
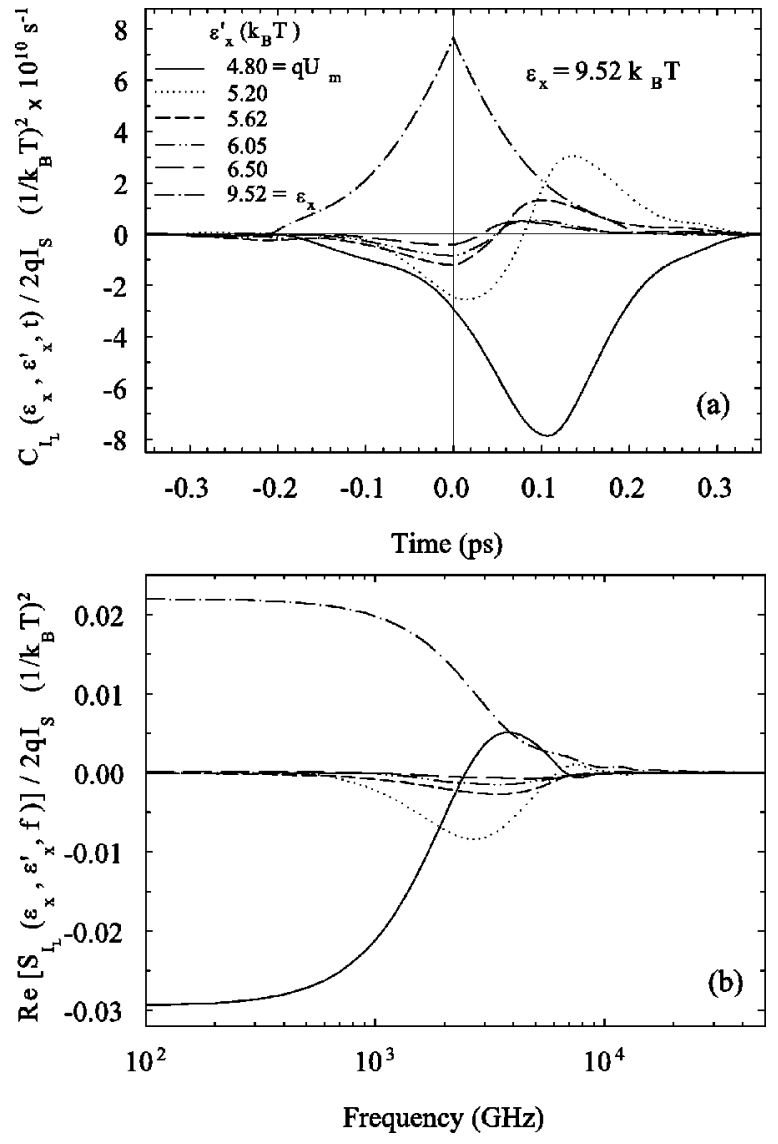

FIG. 5. (a) Correlation functions between current fluctuations due to electrons injected with energies $\varepsilon_{x}$ and $\varepsilon_{x}^{\prime}$ and (b) real part of the corresponding spectra for the case of $\varepsilon_{x}=9.52 k_{B} T$ and several values of $\varepsilon_{x}^{\prime}$.

Up to now we have considered just the low-frequency case, in which the only carriers whose transmission is affected by the barrier fluctuations are those injected with $\varepsilon_{x}$ $\approx q U_{m}$ and, accordingly, this is the only energy level exhibiting the influence of Coulomb correlations. However, at high frequencies other correlations can be expected. Indeed, even if the carriers injected at the left contact with $\varepsilon_{x}$ $>q U_{m}$ are completely transmitted to the right one, their dynamics is affected by the barrier fluctuations. This influence is more significant the closer $\varepsilon_{x}$ is to $q U_{m}$, since this implies that electrons pass over the barrier more slowly. Therefore, the carriers injected with $\varepsilon_{x}$ slightly higher than $q U_{m}$ are the main candidates to exhibit correlations at high frequencies. To confirm this conjecture, in Fig. 5 we report $C_{I_{L}}^{d}\left(\varepsilon_{x}, \varepsilon_{x}^{\prime}, t\right)$ [see Fig. 5(a)] and $\operatorname{Re}\left[S_{I_{L}}^{d}\left(\varepsilon_{x}, \varepsilon_{x}^{\prime}, f\right)\right]^{19}$ [see Fig. 5(b)] for several values of $\varepsilon_{x}^{\prime} \geqslant q U_{m}$ and $\varepsilon_{x}=9.52 k_{B} T$. From Fig. 5(b) we observe that, even if at low frequency only the autocorrelation $\left(\varepsilon_{x}=\varepsilon_{x}^{\prime}\right)$ and the cross correlation with carriers at $\varepsilon_{x}^{\prime} \approx q U_{m}$ are different from zero, at high frequencies there exist significant correlations with carriers injected at energies above the barrier. In Fig. 5(a) the shape of the $C_{I_{L}}^{d}\left(9.52 k_{B} T, \varepsilon_{x}^{\prime}, t\right)$ curves can be explained as follows. When a positive fluctuation of $I_{L}\left(9.52 k_{B} T, t\right)$ takes place (excess of carriers injected at $9.52 k_{B} T$ passing over the bar- 
rier), the electrons at $\varepsilon_{x}^{\prime}>q U_{m}$ are slowed down due to the increase of the barrier amplitude. As a consequence, we observe the onset of a negative fluctuation of $I_{L}\left(\varepsilon_{x}^{\prime}, t\right)$ which leads to negative values of $C_{I_{L}}^{d}\left(9.52 k_{B} T, \varepsilon_{x}^{\prime}, t\right)$ at the shortest times. Once the excess carriers injected at $9.52 k_{B} T$ are far away from the barrier, this decreases in amplitude and electrons with $\varepsilon_{x}^{\prime}>q U_{m}$ become faster when passing over it, thus leading to positive fluctuations of $I_{L}\left(\varepsilon_{x}^{\prime}, t\right)$ [i.e., positive values of $C_{I_{L}}^{d}\left(9.52 k_{B} T, \varepsilon_{x}^{\prime}, t\right)$ at longer positive times]. This effect is more pronounced and occurs for longer times the closer $\varepsilon_{x}^{\prime}$ is to $q U_{m}$, since the injection rate is higher and the carriers are slower. As a result of this behavior, $\operatorname{Re}\left[S_{I_{L}}^{d}\left(\varepsilon_{x}, \varepsilon_{x}^{\prime}, f\right)\right]$ exhibits negative values with a minimum of larger amplitude, appearing at lower frequencies, the smaller $\varepsilon_{x}^{\prime}$ is. In the case of the cross correlation with the carriers injected at $\varepsilon_{x}^{\prime} \approx q U_{m}, C_{I_{L}}^{d}\left(9.52 k_{B} T, q U_{m}, t\right)$ takes only negative values, since these carriers, instead of being initially slowed down and then accelerated, are just reflected back to the contact.

\section{CONCLUSIONS}

In this paper we have investigated the role played by correlations between current fluctuations due to carriers with different energy on the Coulomb suppression of shot noise in a semiconductor ballistic diode connected to two degenerate thermal reservoirs. To this purpose, we have carried out a decomposition of the current autocorrelation function and of the corresponding spectral density into the contributions coming from the correlations between groups of carriers pertaining to different energies. Such a procedure has been implemented in a self-consistent Monte Carlo simulator. The numerical results show that Coulomb suppression at low frequency is controlled by the negative correlation existing between current fluctuations due to the electrons injected with an energy close to that of the potential barrier present in the active region of the diode and those due to all other electrons injected with higher energies. Remarkably, electrons around the energy of the potential barrier are found to be strongly autocorrelated at low frequency. We have also evidenced the presence of correlations at high frequency, where, in contrast with the low-frequency case, carriers well above the barrier display significant correlations. The recent development of electron devices based on ballistic structures with different geometries $^{2,4,5,20}$ is promising to find out an experimental validation of the present results.

\section{ACKNOWLEDGMENTS}

The authors are grateful to O. M. Bulashenko and V. A. Kochelap for stimulating discussions on the subject. Partial support from the Ministerio de Ciencia y Tecnología (Spain) and FEDER through Projects No. TIC2001-1754, TIC200204280-C03-01 and the Ramón y Cajal program, from the Consejería de Educación y Cultura de la Junta de Castilla y León through the project No. SA057/02, and from the ItalySpain Joint Action of the MIUR Italy (Ref. IT-109) and MCyT Spain (Ref. HI2000-0138) is gratefully acknowledged.
${ }^{1}$ F.G. Pikus and K.K. Likharev, Appl. Phys. Lett. 71, 3661 (1997).

${ }^{2}$ I. Shorubalko, H.Q. Xu, I. Maximov, P. Omling, L. Samuelson, and W. Seifert, Appl. Phys. Lett. 79, 1384 (2001).

${ }^{3}$ J.H. Rhew, Z. Ren, and M.S. Lundstrom, Surf. Sci. Spectra 46, 1899 (2002).

${ }^{4}$ K. Hieke and M. Ulfward, Phys. Rev. B 62, 16727 (2000).

${ }^{5}$ A.M. Song, P. Omling, L. Samuelson, W. Seifert, I. Shorubalko, and H. Zirath, Jpn. J. Appl. Phys. 40, L909 (2001).

${ }^{6}$ Y. Naveh, A.N. Korotkov, and K.K. Likharev, Phys. Rev. B 60, R2169 (1999).

${ }^{7}$ T. González, O.M. Bulashenko, J. Mateos, D. Pardo, and L. Reggiani, Phys. Rev. B 56, 6424 (1997).

${ }^{8}$ T. González, J. Mateos, D. Pardo, and L. Reggiani, Physica B 272, 285 (1999).

${ }^{9}$ O.M. Bulashenko, J.M. Rubí, and V.A. Kochelap, Phys. Rev. B 61, 5511 (2000).

${ }^{10}$ O.M. Bulashenko, J.M. Rubí, and V.A. Kochelap, Phys. Rev. B 62, 8184 (2000).

${ }^{11}$ O.M. Bulashenko and J.M. Rubí, Phys. Rev. B 64, 045307 (2001)

${ }^{12}$ O.M. Bulashenko and J.M. Rubí, Phys. Rev. B 66, 045310 (2002).
${ }^{13}$ G. Gomila, I.R. Cantalapiedra, T. González, and L. Reggiani, Phys. Rev. B 66, 075302 (2002).

${ }^{14}$ T. González, J. Mateos, D. Pardo, L. Varani, and L. Reggiani, Semicond. Sci. Technol. 14, L37 (1999).

${ }^{15}$ L.S. Levitov and G.B. Lesovik, Pis'ma Zh. Éksp. Teor. Fiz. 58, 225 (1993) [JETP Lett. 58, 230 (1993)].

${ }^{16}$ Ya.M. Blanter and M. Buttiker, Phys. Rep. 336, 1 (2000).

${ }^{17}$ O. M. Bulashenko, J. M. Rubí, and V. A. Kochelap, in Statistical and Dynamical Aspects of Mesoscopic Systems, edited by D. Reguera, G. Platero, L. L. Bonilla, and J. M. Rubí (Springer, Berlin, 2000), p. 343.

${ }^{18}$ Actually, for convenience of the simulation scheme, it is the longitudinal wave vector $k_{x}$ which is discretized into 50 uniform meshes that become nonuniform when transformed into energy intervals.

${ }^{19} \operatorname{Im}\left[S_{I_{L}}^{d}\left(\varepsilon_{x}, \varepsilon_{x}^{\prime}, f\right)\right]$ does not contribute to $S_{I}^{d}(f)$, since $\operatorname{Im}\left[S_{I_{L}}^{d}\left(\varepsilon_{x}, \varepsilon_{x}^{\prime}, f\right)\right]=-\operatorname{Im}\left[S_{I_{L}}^{d}\left(\varepsilon_{x}^{\prime}, \varepsilon_{x}, f\right)\right]$ and these terms balance each other when performing the integration in Eq. (9).

${ }^{20}$ J. Mateos, B.G. Vasallo, D. Pardo, T. González, J.S. Galloo, Y. Roelens, S. Bollaert, and A. Cappy, Nanotechnology 14, 117 (2003). 